Document downloaded from:

http://hdl.handle.net/10251/165282

This paper must be cited as:

Colucci, D.; Prats-Montalbán, JM.; Ferrer, A.; Fissore, D. (2021). On-line product quality and process failure monitoring in freeze-drying of pharmaceutical products. Drying Technology. 39(2):134-147. https://doi.org/10.1080/07373937.2019.1614949

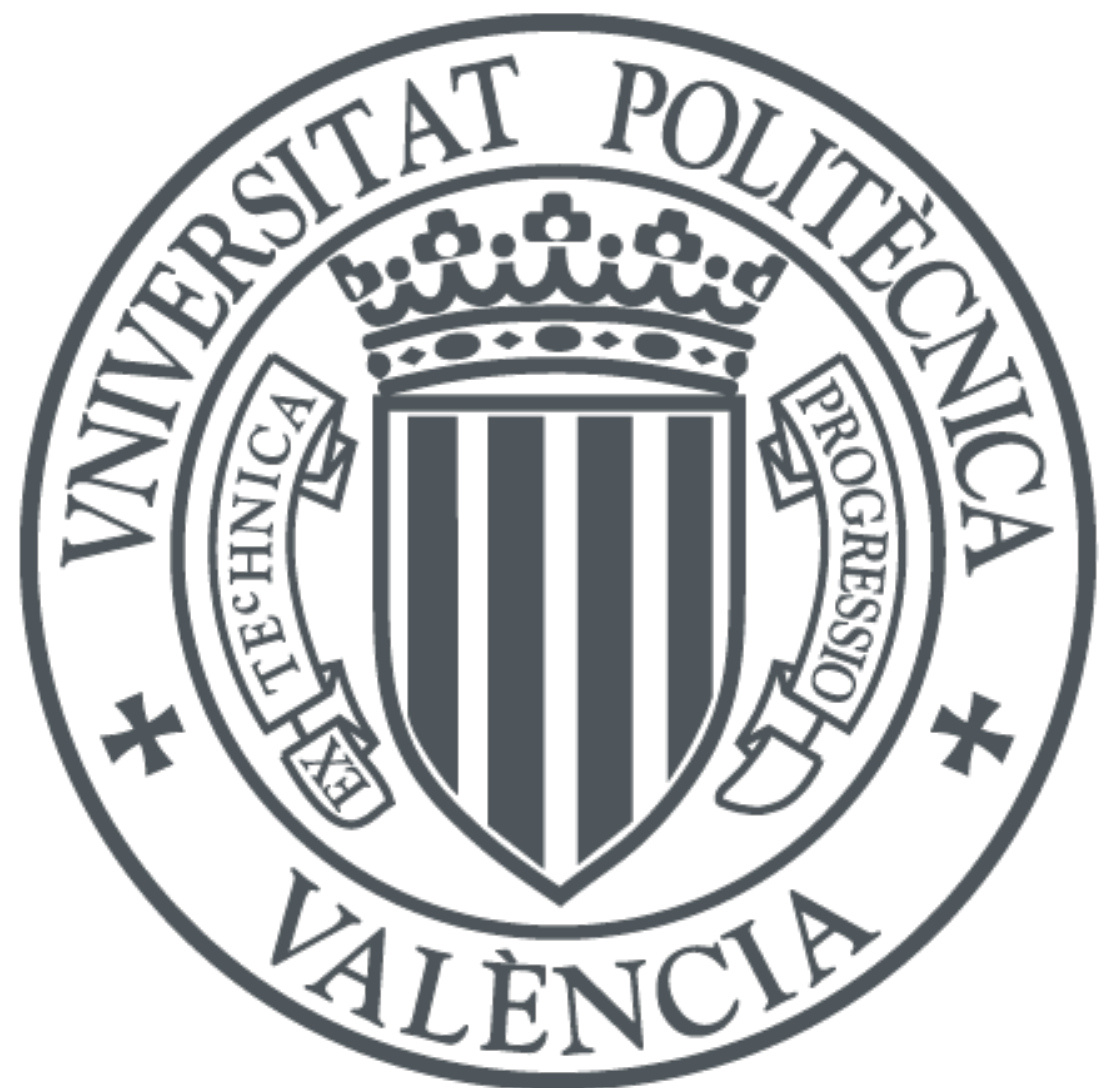

The final publication is available at

https://doi.org/10.1080/07373937.2019.1614949

Copyright Taylor \& Francis

Additional Information

This is an Author's Accepted Manuscript of Domenico Colucci, José M. Prats-Montalbán, Alberto Ferrer \& Davide Fissore (2021) On-line product quality and process failure monitoring in freeze-drying of pharmaceutical products, Drying Technology, 39:2, 134-147, DOI: 10.1080/07373937.2019.1614949 [copyright Taylor \& Francis], available online at: http://www.tandfonline.com/10.1080/07373937.2019.1614949 
Submitted for publication on

Drying Technology

\section{On-line product quality and process failure monitoring in freeze- drying of pharmaceutical products}

In this work the information provided by a non-invasive imaging sensor was used to develop two algorithms for real time fault detection during the Vacuum FreezeDrying of single dose pharmaceuticals. Two algorithms based on multivariate statistical techniques, namely Principal Component Analysis (PCA) and Partial Least Square Regression (PLS), were developed and compared. Five batches obtained under Normal Operating Conditions (NOC) were used to train a reference model of the process; the classification abilities of these algorithms were tested on five more batches simulating different kind of faults. Good classification performances have been obtained with both the algorithms. Coupling the information obtained from an infrared camera with that of other variables obtained from the PLC of the equipment and from the textural analysis performed on the RGB images of the product, strongly improves the performances of the algorithms. The proposed algorithms can account for the heterogeneity of the batch and aim to reduce the off-specification products.

Keywords: infrared imaging; process monitoring; multivariate statistical process control; freeze-drying. 


\section{Introduction}

Water, or more generally solvent, removal is a mandatory step in the preparation of many pharmaceutical products, since the mobility of the molecules in the liquid state enhances the kinetics of many chemical and physical degradation processes. Trapped into a solid structure, the active compounds could be stored, also at room temperature, for long time. Freeze-drying is particularly appreciated, compared to other drying processes, since the water content is reduced at low temperature, via sublimation from a frozen product, thus avoiding any damage to thermolabile molecules [1-4].

The operation is mostly a batch process and a typical freeze-drying cycle is composed of three stages, namely freezing, primary drying, and secondary drying. The liquid product is first poured into single dose vials, more rarely into trays, and placed on the shelves of the drying chamber. A technical fluid removes heat from the product until the free solvent freezes, while a certain amount of the solvent remains bounded to the solid structure, in a liquid state. The cooling rate will determine the dimensions of the ice crystals and, thus, of the pores inside the dried layer of the product, which directly affects the mass transfer resistance to vapor flux in the drying stages. Once the product is completely frozen, the pressure in the chamber is reduced and the shelf temperature is raised to allow the sublimation of the ice. As the water vapor leaves the product the ice front (the interface separating the frozen layer from the dried one) recedes from the top to the bottom of the product. In the secondary drying stage, the bound water is removed to reach the desired value of residual moisture [1-5]. This is achieved by increasing the temperature in the chamber; in most cases, pressure does not have a real effect on this step [6].

The US Food and Drug Administration Guidance for industry PAT [7] strongly encourages the development of Process Analytical Technologies (PAT) for on-line 
monitoring and control of the evolution of the process and obtain the desired quality of the final product in the whole batch.

Several reviews on the available PAT for monitoring of the vacuum freeze-drying process can be found in the literature [8-11]. One of the most recent one is that of Fissore et al. [12], in which the technologies proposed for process monitoring and control were compared and their pros and cons were discussed. The monitoring systems reviewed where basically divided into four groups according to the measured variables: product temperature, heat flux, sublimation flux and other variables. Some of these technologies, especially among those measuring the mass or heat flux inside the product, generally provide an estimation of the average value of the variables of interest in the whole batch. Therefore, it is impossible to account for the in-batch heterogeneity basically due to thermal radiation from the chamber walls and mass flow dynamics [13]. Also, uncontrolled nucleation, due to the stochastic nature of freezing, may create non-uniformly distributed pores, i.e. differences in the mass transfer. Different methodologies for a controlled nucleation have been presented [14].

Measuring the temperature of the product in different position inside the batch, the lack of homogeneity could be easily handled [15-16]. The main drawback of this approach is that thermocouples could interfere with the drying kinetics of the process and the presence of electronics and wires does not fit the requirements of the pharmaceutical industries in terms of automatization (i.e. loading, stoppering and discharge) and sterility. Aiming to come with these issues, Grassini et al. proposed plasma sputtered sub-micrometric temperature probes embedded into the vial wall [17], while Emteborg et al. [18] firstly proposed to measure the temperature of the product using a thermal camera. In their work the sensor was mounted on the roof of the equipment and a hole was made on the chamber roof to allow a free view of the product on the top shelf. In this solution the camera is protected from the harsh 
characteristics of a freeze-drying chamber, namely low temperature, low pressure and moisture reach environment, but the position of the camera, perpendicular to the top shelf, allows only the measurement of the temperature on the top of the vials. Lietta et al. [19] presented and validated a sensor in which the infrared and a RGB camera are enclosed into a case designed to protect the electronics. This design allows positioning the camera inside the drying chamber, with no modifications of the existing equipment, and to measure the temperature on the wall of the vials in any shelf. Glass is mostly infrared opaque, but the thermal resistance of the glass wall is normally very low, and the temperature of the glass is only slightly different from that of the product [20].

In this work we focus on primary drying, the most demanding stage of the entire process because of the higher water content, and in the prompt detection any abnormal variation of the process conditions from those that could guarantee the desired product quality. A quick and reliable detection of the eventual faults occurring during the process could allow correcting them before the whole batch is impaired reducing both the failure rate and the waste production. If the product is proved to be obtained from a process under statistical control, the number of assay that must be performed, thus the time required, to release the batch could be strongly reduced. Furthermore, since primary drying of a pharmaceutical drug can last many days or even weeks, it is important to determine if a batch is out of its design space and can be stopped before more time is wasted.

The information extracted from the thermal and RGB images of the product was coupled with that of some additional variables obtained directly from the PLC of the equipment to build a model of the design space of the process, accounting for the natural variability of the system, and the control charts able to determine whether the evolution of the process is consistent or not. The amount and kind of the data at hand (i.e. highly redundant, 
noisy and strongly correlated, together with the batch nature of the process, that is, all the variables change with time) requires multivariate statistic techniques to be used.

Two Multivariate Statistical Process Control (MSPC) algorithms, one based on Principal Component Analysis (PCA) [22,23] and the other based on the Partial Least Square Regression (PLS) algorithm [24,25], are presented for real time process monitoring and fault detection. Kourti [26] discussed the relevant role of multivariate statistic techniques in the development of PAT for the pharmaceutical industries. A critical evaluation of many possible approaches to the MSPC of batch processes can be found in Van Sprang et al. [27]. Rato et al. [28][28] recently presented a systematic methodology to compare batch process monitoring methods and compared different approaches in terms of detection strength and speed.

The application of Multivariate Statistical techniques to the processing and the extraction of information from images falls inside the framework of the Multivariate Image Analysis (MIA)[30]. An exhaustive review of the MIA techniques and possible application was presented by Prats-Montalbán et al. [30], while Duchesne et al. [32] reviewed the industrial application of MIA.

The paper is organized in four sections: section 2 introduces some details about the experimental apparatus and the MSPC algorithms, section 3 presents the main results and section 4 the general conclusions of this work.

\section{Materials and methods}

\subsection{Experimental study}

The drying experiments were carried out in lab scale equipment: LyoBeta $25^{\mathrm{TM}}$ freeze-dryer (Telstar, Spain) having a chamber volume of $0.2 \mathrm{~m}^{3}$, a total shelf area of $0.5 \mathrm{~m}^{2}$ 
and a capacity of the external ice condenser of $40 \mathrm{~kg}$. A SCADA system allows measuring and controlling both the temperature of the technical fluid flowing inside the shelves and the chamber pressure. The temperature of the technological fluid cooling the condenser is also measured and acquired in real time. Chamber pressure is measured by two sensors embedded in the equipment, a conductive Pirani gauge (Pirani type PSG-101-S, Inficon, Bad Ragaz, Switzerland) and a capacitive Baratron manometer (Baratrontype 626A, MKS Instruments, Andover, USA). The pressure in the chamber is controlled, acting on the valve between the vacuum pump and the condenser, according to the measurement of the Baratron gauge.

The evolution of the product was monitored using the sensor described by Lietta et al. [19]. It consists of a case in Ertacetal C, designed (IMC Services s.r.l., Italy) to resist and protect the electronics from the temperature, pressure and humidity typical of a drying chamber, hosting an infrared camera (FLIR A35) and a HDTV RGB camera. The data acquired are stored into a microprocessor that can be accessed via wi-fi in real time through a graphical user interface.

The thermal images obtained are 256x320 pixels, and before their analysis they were always corrected for the barrel effect induced by the lens [33] and registered to compensate the minor movement of the vials and the sensor due to the vibration of the equipment [34]. In the following these steps were referred as image pretreatment.

In all tests ten vials (ISO 8362-1 10R) were placed at $30 \mathrm{~cm}$ from the sensor, and a new image was acquired every five minutes. A reference model of the process was created on the data obtained processing five batches. Each vial was filled with $5 \mathrm{ml}$ of a solution $10 \%$ b.w. of sucrose (Sigma Aldrich, $99.5 \%$ ) at $-20^{\circ} \mathrm{C}$ and $20 \mathrm{~Pa}$. These data set constitutes our normal operating conditions (NOC) data set.

The detection ability of the system was evaluated in five additional batches. In batch 7 
a failure in the vacuum system has been simulated: after 5 hours of drying, chamber pressure was raised to $50 \mathrm{~Pa}$. In batch 8 the shelf temperature was set to $-10^{\circ} \mathrm{C}$ while in batch $10 \mathrm{a}$ solution 5\% b.w. of sucrose was used. Batch 6 and 9 aimed to prove the ability of the model to detect faults affecting the single vials, while shelf temperature and chamber pressure were set to the NOC values. In batch 6, due to the vibrations of the equipment, one vial felt down, while, in batch 9, only four vials were filled with a $10 \%$ solution. A piece of glass was inserted into two of the remaining vials, one was filled with pure water, one with the same $5 \%$ b.w. solution used for batch 10 and the remaining two with respectively 2.5 and $7.5 \mathrm{ml}$ of solution. The test set used to evaluate the monitoring performances of the algorithms was completed with five NOC batches obtained randomly sampling the single vials from the 5 batches of the training set. Merging the measurements from different vials in a new batch creates a new correlation structure inside the data, thus a realistic approximation of a new NOC batch. Table 1 resumes the drying experiment performed, the operating conditions tested, and the kind of fault considered. It also reports whether the single batch was used to train the reference models or to test them.

\subsection{Multivariate Statistical Process Control (MSPC)}

A $10 \mathrm{R}$ vial at $30 \mathrm{~cm}$ from a thermal camera has a diameter of approximately 22 pixels. $5 \mathrm{ml}$ of solution correspond to approximately a height of the liquid inside the vial of 20 pixels. Thus, the portion of the image corresponding to the product into every vial has an area of approximately 400 pixels. This region was segmented [35] and the temperature distributions of these areas were extracted for all the ten vials of each one of the 500 images of a single batch. Given the huge amount of data, these thermal profiles have been characterized to study their evolution: mean, standard deviation (std), skewness and kurtosis 
were extracted from each area. An example and a discussion about the features extracted from the thermal images can be found in Colucci et al. [35].

The obtained trajectories were aligned removing the images acquired before the sublimation started. The variability among the vials within the same batch, that is the effect of the position of the vial on the shelf, was included among the modeled variables, modeling mean, standard deviation (std), skewness and kurtosis for each one of the ten positions that a vial could occupy on the shelf. The results were collected into a three-dimensional data array $\underline{\mathbf{X}}(I \times J \times K)$, a data structure typical of a batch process, where $I$ is the number of batches (i.e. five), $K$ is the total number of image acquisitions (five hundred), while the variables $(J)$ are forty.

Multivariate statistical process control algorithms were created using both Principal Component Analysis (PCA) and Partial Least Square (PLS) [23;25;36]. Both algorithms can only deal with bi-dimensional matrices. Thus, the original 3D data structure was batch wise unfolded (BWU), that is the features extracted for a single observation were put beside each other in order of time acquisition, to obtain a matrix $\mathbf{X}(I \times J K)$ [37]. Camacho et al. [38] proved that this kind of unfolding is the best approach for problems of batch process monitoring. The reference PCA and PLS models were calculated on the feature extracted from the batches 1 to 5 ; in all cases the $\mathbf{X}$ matrix was mean centered and scaled to unitary variance to give to all the variables the same a priori relevance to the models.

A PCA model is a linear decomposition of a data structure:

$\mathbf{X}=\mathbf{T} \cdot \mathbf{P}^{\prime}+\mathbf{E}$

where $\mathbf{T}$ is the $I \times A$ score matrix, $\mathbf{P}$ is the $A \times J K$ loading matrix and $\mathbf{E}$ is the residual matrix, 
having the same dimension of the original matrix $\mathbf{X}$. A is the number of principal components extracted.

A PLS model with A principal components has the following structure:

$$
\begin{aligned}
& \mathbf{X}=\mathbf{T} \cdot \mathbf{W}^{*} \cdot \mathbf{E} \\
& \mathbf{Y}=\mathbf{U} \cdot \mathbf{C}^{\prime}+\mathbf{F}
\end{aligned}
$$

where $\mathbf{T}$ is the $I \times A$ score matrix of $\mathbf{X}$ and $\mathbf{U}$ is the $I \times A$ score matrix of $\mathbf{Y}, \mathbf{W}^{*}$ is the $A \times J K$ loading matrix of $\mathbf{X}$ and $\mathbf{C}$ is the corresponding $A \times J^{*}$ loading matrix of $\mathbf{Y} . \mathbf{E}$ and $\mathbf{F}$ are the matrices of the residuals, having the same dimension respectively of the matrices $\mathbf{X}$ and $\mathbf{Y}$. The matrix $\mathbf{Y}$ was mean centered and scaled to unitary variance.

The main difference between the PCA and PLS is that PLS creates a regression model between a matrix $\mathbf{X}$, in this case the same used in the PCA algorithm, and a matrix of quality variables $\mathbf{Y}\left(I \times J^{*}\right)$, where $I$ is again the number of observations in the NOC data set, and $J^{*}$ the number of quality variables considered. With this approach we are not only looking for unexpected deviation in the evolution of the process variables, but also for their effect on the final quality of the product, which is our real interest [39]. Quality variable in this framework could be everything, e.g. protein activity, rehydration rate, impurities and so on. In this work we used the values of onset $\left(t_{1}\right)$ and offset $\left(t_{1}\right)$ read from the Pirani Baratron ratio, that is the ending time of primary drying [40], and the average temperature at the bottom of the vials measured at the beginning of the sublimation stage $\left(T_{0}\right)$, at time $t_{1}\left(T_{1}\right), t_{2}\left(T_{1}\right)$ and at the end of the primary drying $\left(T_{\text {end }}\right) . T_{0}, T_{1}, T_{2}$ and $T_{\text {end }}$ were obtained directly from the thermal images; Liotta et al. [19] proved that these measurements are in good agreement with those obtained with a thermocouple inserted inside the vial. The resulting $\mathbf{Y}$ matrix for all the 15 
batches is reported in Table 2 .

Using the PLS it is possible to obtain a real-time prediction of the final quality attributes of the product, Eq. (4) and, following the procedure presented by Nomikos and McGregor [25], we can also build the control charts of this predicted value:

$$
\mathbf{Y}_{\text {pred }}=\mathbf{X} \cdot\left(\mathbf{W} * \mathbf{C}^{\prime}\right)+\mathbf{F}^{*}
$$

In both PCA and PLS based MSPC a model is created on the data obtained from the NOC data set, then the vector of the new observation, the new batch, is projected into the subspace defined by the latent variables. Here the information is finally compressed into two independent indexes: Hotelling $\mathrm{T}^{2}\left(T^{2}\right)$ and the Instantaneous Square Prediction Error (SPEI) defined by Eq. (5) and Eq. (6):

$$
\begin{aligned}
& T^{2}=\sum_{a=1}^{A} \frac{t_{a}^{2}}{\lambda_{a}} \\
& S P E I=\sum_{c=1+(k-1) J}^{J K} e_{c}^{2}
\end{aligned}
$$

where $t_{a}$ is the $a$-th score and $e_{c}$ is the error obtained after predicting the measurement of variable $c$ for a certain observation. $\lambda_{\mathrm{a}}$ is the eigenvalue of the $a$-th principal component and $k$ the actual time instant at which the measurement is performed.

Using the distributions of these indexes obtained from the training data set, it is possible to calculate the Upper Control Limits (UCL) of the control charts [41]. The control limits obtained were initially calculated taking the 99.5 percentile of the distributions and were then manually retuned to provide the best classification performance. When an 
observation trespasses the UCL the contribution plots can be used to figure out which of the original variables are responsible for this deviation from the evolution of the NOC data. More details about the algorithms and how these statistical techniques can be used to perform online monitoring and fault detection can be found in [42]. For a detailed discussion on the application of these two methodologies and the pros and cons of each one, the reader could refer to [43].

At the generic instant of time $k$ only a part of the new observation is known, so the missing part of the vector must be forecasted in order to allow the projection of the whole vector in the latent variable sub-space [42]. In this work the Trimmed Score Regression (TSR) algorithm developed by Arteaga and Ferrer [44] was used. Details on this algorithm and its application to PCA and PLS based MSPC algorithms can be found respectively in $[45,46]$ and $[47,48]$.

\subsection{Additional variables}

The major advantage of using multivariate statistical techniques is that these algorithms could easily deal with great amount of data, no matter their degree of correlation or the redundancy of the information. It is possible then to add more variables to the matrix $\underline{\mathbf{X}}$ $\left(I \times\left(J+J_{v}\right) \times K\right)$ to better highlight the correlation structure hidden inside the data and ease the classification process.

Five variables $\left(J_{v}=5\right)$ were read, for every batch, in the same time instant the thermal image was acquired and added to the forty already extracted from the thermal images. The PLC of the equipment provides measurements from both the conductive Pirani gauge and a capacitive Baratron manometer. The measurement of the thermal conductivity gauge is affected by the composition of the atmosphere inside the drying chamber. The ratio between 
the two pressure measurements is greater than one when the ice is sublimating, while approaches the unitary value at end of the primary drying [40] and for this reason is a state of art methodology to identify the end point of the primary drying. For every image the corresponding value of the Pirani pressure and the Pirani-Baratron (PB) ratio were collected and added to the model.

From the PLC of equipment, it is possible to obtain the instant values of the temperature of the fluid flowing inside the shelves and of the utilities cooling the condenser wall.

The last variable included is an index obtained from the textural analysis of the RGB images [49]. The emissivity of the glass of the vials is quite high, approximately 0.9 at the temperatures typical of a primary drying, thus it is almost completely opaque to infrared radiation. This means that we are measuring the temperature on the vial wall, not that of the product, and, even if they are quite close to each other, the inclusion of a variable providing a direct look on the product could be useful. Texture, namely fractures, shrinkage, holes, scratches and so on, is deeply related to both mass and heat transfer and can be directly obtained from the RGB images [50-51].

The RGB images are 820x616 pixels and were pretreated and segmented exactly as the thermal ones, see Figure 1, except for the barrel effect correction that was not required. The region corresponding to the product of all the vials was segmented and the skewness of the pixels intensity on a mobile window of $3 \times 3$ pixels was measured. This provides a vector of approximately thirty thousand values for every image whose average was selected as index of the textural characteristics of the product [34]. The skewness is an index of how asymmetrical a statistical distribution is; it is positive when the mass of the data is concentrated on the left, while is negative when it presents a left tail longer than the right one. 
A positive or negative skewness reports the presence of intensity gradients mostly associates with the borders of a fracture, e.g. a scratch or a detachment from the vial typical of the shrinkage of the product. The skewness allows also to filter the effects of an asymmetrical external illumination of the vials.

The information in the two blocks of variables $J$ and $J_{v}$ is different because they depict distinct aspects of the process. Multivariate statistical techniques, when all the variables are unitary scaled, make no difference between them, and it could happen that the noise of some strongly correlated measurements becomes more important than the information, possibly completely different from the previous, but represented only by one single variable. For this reason, the two blocks of variables have been block scaled [52]. Three different block scaling approaches have been compared:

- No block scaling $(\mathrm{N})$ : each one of the forty-five variables count as one;

- Unitary scaling (U): the two blocks have the same, unitary variance;

- Inverse scaling (I): the block obtained from the thermal images is scaled to unitary variance, as each one of the five variables forming the second block.

The classification performances of the algorithms presented in subsection 2.2 were evaluated and compared, for different number of principal components extracted, with and without additional variables and for all the three distinct types of block scaling according to Eq.(7):

$f_{A}=(1-\alpha) \cdot(1-\beta)$

where $\alpha$ is the overall type I error rate (i.e. false positive rate) and $\beta$ is the overall type II error rate (i.e. false negative rate) [53]. A false positive is an NOC batch classified as a fault; a false positive is a fault not trespassing the control limits of the control charts. The lower $\alpha$ and $\beta$ the more $f_{A}$ approaches the unit. 


\section{Results}

Figure 2 presents an example of the control charts obtained for three batches of the test set, namely batches 6,9 and 12 , when a PCA model with two principal components is used. Figure 2A reports the SPEI control charts. Batch 12, gray line, is perfectly discriminated as a NOC batch and lays all the time behind the black thick line of the control limits. Batches 6 and 10, two faults, cross the control limits and are detected as faults by the SPEI control chart. The $T^{2}$ control chart, Figure 2B, correctly discriminates batches 6 and 12 but not batch 9, which is a false negative. Notice that most of the time its $T^{2}$ values are lower than those of batch 12, making it impossible to set apart this two batches retuning the control limits. Also, batch 10 was detected by the SPEI, even if it just mildly overtakes the control limit and normally not for the whole batch, while it was impossible to discriminate it from the NOC batches in the $T^{2}$ control chart.

Figure 3A shows the SPEI control chart for batches 14 (NOC) and 8 (fault) and 9 (fault) while Figure $3 \mathrm{~B}$ shows the $T^{2}$ control chart for the same observations when a PLS model with two principal components was built. Both the $T^{2}$ and SPEI control charts correctly classify batches 8 and 14. Batch 9 was correctly classified by the SPEI but not from the $T^{2}$ control chart.

Figure $4 \mathrm{~A}$ and $4 \mathrm{~B}$ reports the control charts obtained on the quality variables of for batches 8 and 13. In the first case almost all the 6 quality variables match the expected value. or at least they are included inside the $99.5 \%$ upper and lower control limits. In the second case five of the variables are mispredicted and should call the attention. Furthermore, in this case, we cannot say if they fall inside the upper and lower bound, because, if the $T^{2}$ and/or the SPEI fall outside their UCL, the model is no more reliable and so are the control limits obtained for the predicted $y$. For this reason, they have not been reported for batch 8 . 
Figure 5 shows a comparison of the fault detection abilities of the MSPC based algorithms developed in this study. The PLS presents two false negatives less in the $T^{2}$ control chart than the PCA when only one principal component is extracted. They touch when two PC are used, and twist when three latent variables are used, having the PCA mildly overperforming the PLS. They all show and increasing trend until the unitary value is reached for a model using four principal components.

In all the simulations performed $\alpha$ was zero, i.e. no NOC batches were reported as faults. All the errors of classification were due to false negatives and only in the $T^{2}$. SPEI always correctly classified all the ten batches, also when only one principal component was used, and this is true for both PCA and PLS.

Camacho et al. [54] already discussed this lower reliability of the Hotelling $T^{2}$ when dealing with data from a batch process. This is due to the fact that, while the SPEI is measured, according to Nomikos and MacGregor [42] on the variables measured at the present time, $T^{2}$ statistics condenses the information that all the variables, those already measured form the beginning and those forecasted according to the reference model, provide to the scores of the observations. If the matrix has been BWU most of the variables are the same measurement at different instant of time. This creates a strong auto-correlation inside this statistic that makes more difficult to overtake of the control limits. Only batches 6 and 8 are always trespassing the UCL in the $T^{2}$ control charts. In batch 6 one vial felt down after one hour from the beginning of the process. The temperature distribution characterized by the algorithm from that moment is that of the door of the camera, higher and basically constant during the process. In batch 8 the temperature of the fluid was set $10{ }^{\circ} \mathrm{C}$ above the set point of the NOC data set. Everything was warmer inside the chamber, also the vials. In these two cases the breakage of the correlation structure is strong enough to appear abnormal in both 
the control charts, even if the less reliable one is also slower in detecting the fault. SPEI detects the fall of the vial soon after it happens while the $T^{2}$ of batch 6 needed more than one hours to cross the control limits [35].

This remark led to the idea that more information we can provide to the model the clearer will be the eventual breakage in the correlation structure, and, if the information included into the thermal images is not enough, we should include more variables. This also serves a higher goal. Including information from all the other sensors in the equipment allow us to monitor the whole equipment and the behavior of the control loops for pressure and shelves temperature or the condenser cooling system.

Figure 6 shows the profiles of the five additional variables measured for all the batches; the numbers reported above refer to the misbehaving batch.

The temperature of the fluid inside the shelves, Figure 6A, reaches the set point after a short overshoot and, in all cases, it is well controlled with low variability in the measurements. The condenser temperature is not expected to provide any real information, since all the profiles appear to be superimposed and the noise of the measurement will probably cover any slightly difference. Larger batches could show a greater effect on this variable. Batch 7 shows an anomalous trend in both Pirani and the Pirani-Baratron profiles. Batch 10 appears to have a mildly strange PB ratio.

Figure 7A and 7B are a good example of how a texture analysis performed on the RGB images could be complementary to the information obtained from the thermal ones. Batch 7 has been detected as an outlier also when only the thermal information was used, thus the thermal profiles are abnormal, but Figure 7A does not show any distinctive feature and the UCL were only slightly trespassed. But, inside the vials, after the pressure was raised, the product boiled, creating many clearly visible holes inside the cake, Figure $7 \mathrm{~B}$, that moved 
the average skewness well beneath the zero. In batch 9 the vial filled with pure water, as well as those containing a piece of glass or the one with a lower volume of solution, increase the global skewness and, although the measurement is averaged on all the ten vials, also the single fallen vial in batch 6 has been detected, see Figure 7C.

The additional variable strongly improved the classification performance of both the algorithms, see Figure 8 . In both cases the value of $f_{A}$ for the models obtained with the $\mathrm{N}$ approach, i.e. the model uses the additional variables, but the two blocks are not scaled (black squares), are always superior or the same as those of the model built with only the thermal features. A strong improvement of the classification performances can be obtained with the appropriate block scaling of the variables. The I wise block scaling approach, followed by the unitary scaling (U) approach gave the better performances.

The fact that in all cases, no matter the number of variable used, the algorithm or the block scaling approach, when four PC are used $f_{A}$ becomes unitary, should warn about the possibility of an overfitting. Four is the maximum number of principal components that can be extracted with five mean centered observations, thus, including also the less important, we model all the possible information, including the noise. On a little training set like the one used for this work, modeling this residual variance could give the false idea that the monitoring performances really improve, but including the noise in the model relaxes the UCL and results into less responsive control charts. To avoid this problem the model should be built on larger data sets and validated using other and more NOC observation.

In any case, a two principal components MSPC model with inverse or unitary scaled blocks should provide the required performance with a certain reliability in all cases.

The performance index presented by Eq. (7) does not care about the quality of the prediction. A second approach that could be used to determine the number of principal 
components to be extracted in a PLS model, is to look for the one maximizing the prediction ability of the model since this would automatically guarantee a good classification on the basis of the quality variables control charts. The most accepted criterion to maximize the prediction ability of a PLS model is to look for the maximum cross-validation index $Q^{2}$ [55]. Figure 9 shows the evolution of the $Q^{2}$ index for the first three components of all the different PLS models obtained. The variability inside the NOC data set is quite high, since, in the best case only a $26 \%$ of the variability of the $\mathbf{Y}$ matrix composed by the five NOC batches could be explained. In all case the $Q^{2}$ increases as the number of PC is increased. The $Q^{2}$ increases as the additional variables are added and weighted to become more important when two or more principal components are extracted. The cross-validation index should present a maximum for a certain number of principal components due to the reduction of the prediction performances when the noise starts to be modelled. No one of the cases shows this maximum which could be a symptom of the lack of observations.

In general, we could state that including more variables improves both the fault detection performances of the algorithms and the quality of the prediction and of the monitoring on the product quality variables. The kind and number of the quality variables at hand, the precision of the measurement obtained and their variability are the key features in selecting the best algorithm and data pretreatment approach to be used [40].

\section{Conclusions}

In this work we present a process analytical technology able to address the problem of the real-time fault detection in a vacuum freeze-drying process aiming to guarantee the quality of the whole batch. The first issue of every monitoring system is the acquisition of 
data from the process and this has been a strong limit in case of many pharmaceutical processes because of the sterility requirements and the sensitivity of the products processed. In this paper we used the information obtained from a thermal and a RGB camera to monitor the evolution of the process and to detect any deviation of the batch from its design space without interfere with the process or with the dynamics of the product.

Multivariate statistical techniques like PCA and PLS can deal with large amount of low quality (noisy, auto and cross-correlated etc.) data to develop algorithms able to detect in real time any kind of fault inside the drying chamber. A large set of possible faults was simulated, error in the control loops for the shelf temperature or the chamber pressure as well as errors in the solid content of the solution or the volume of solution filled and used to test these algorithms. The results obtained show that at least one of the two control charts, the SPEI, performs in an excellent way in all cases and can classify all faults independently of its nature. Coupling the feature extracted from the thermal images with the variables acquired by the PLC of the equipment, and a textural index extracted from the RGB images, it is possible to improve the performance of the system and obtain a more reliable classification of the observations.

The kind of algorithm to be used, the number and kind of variables to model and the data pretreatment depend on the specific problem, providing also a certain degree of freedom in the optimization of the MSPC algorithm. Indeed, these results should be validated on batches with a larger number of vials and for more consistent data sets.

In future works, these techniques of Multivariate Image Analysis, especially the PLS, could be used to derive input-output statistical models of the process for prediction, control and optimization of the process. 


\section{Acknowledgement}

The authors would like to acknowledge Elena Lietta for her support in the experimental investigation. 


\section{References}

[1] Mellor, J.D. Fundamentals of freeze-drying. Academic Press: London, 1978.

[2] Jennings, T.A. Lyophilization: introduction and basic principles; Interpharm/CRC Press: Boca Raton, 1999.

[3] Oetjen, G.W.; Haseley P. Freeze-Drying. Wiley-VHC: Weinheim, 2004.

[4] Fissore, D., 2013. Freeze-drying of pharmaceuticals, in: Swarbrick J (Ed.), Encyclopedia of Pharmaceutical Science and Technology, 4th Edition. CRC Press, London, pp. 1723-1737.

[5] Costantino, H.R.; Pikal, M.J. Lyophilization of Biopharmaceuticals; AAPS Press: Arlington, VA, 2004.

[6] Pikal, M.J., Shah, S., Roy, M.L., Putman, R. The secondary drying stage of freeze drying: drying kinetics as a function of temperature and chamber pressure. Int. J. Pharm. 1990, 60(3), 203-207.

[7] U. S. Department of Health and Human Services, Food and Drug Administration, Center for Drug Evaluation and Research (CDER), Center for Veterinary, Medicine (CVM), Office of Regulatory Affairs (ORA), Pharmaceutical CGMPs. September 2004. Guidance for Industry, PAT - A Framework for Innovative Pharmaceutical Development, Manufacturing, and Quality Assurance, 2004. https://www.fda.gov/downloads/drugs/guidances/ucm070305.pdf (last access date: January 2019).

[8] Barresi, A. A.; Pisano, R.; Fissore, D.; Rasetto, V.; Velardi, S. A.; Vallan, A.; Parvis, M.; Galan, M. Monitoring of the Primary Drying of a Lyophilization Process in Vials. Chem. Eng. Process. 2009, 48(1), 408-423.

[9] Johnson, R. E.; Giesler, H.; Teagarden, D. L.; Lewis, L. M. Analytical Accessories for Formulation and Process Development in Freeze-Drying. Am. Pharmaceut. Rev. 2009, 12(5), 54-60.

[10] Wiggenhorn, M.; Winter, G.; Presser, I. The current state of PAT in freeze-drying. Am. Pharmaceut. Rev. 2009, 8(1), 38-44 (European Pharmaceutical Review 2005, 10(4): 87-92). 
[11] Patel, S. M.; Pikal, M. J. Process Analytical Technologies (PAT) in Freeze-Drying of Parental Products. Pharm. Dev. Technol. 2009, 14(6), 567-587.

[12] Fissore, D.; Pisano, R.; Barresi, A.A. Process analytical technology for monitoring pharmaceutical freeze-drying - A comprehensive review. Drying Technol. 2018 In press. doi: 10.1080/07373937.2018.1440590.

[13] Barresi, A.A.; Pisano, R.; Rasetto, V.; Fissore, D.; Marchisio, D. L. 2010. Modelbased monitoring and control of industrial freeze-drying processes: effect of batch nonuniformity. Drying Technol. 2010, 28(5), 577-590.

[14] Pisano, R.; Fissore, D.; Barresi, A. A. Intensification of freeze-drying for the pharmaceutical and food industry. In: Modern drying technology Vol. 5: Process Intensification; Tsotsas E. and Mujudar A.S.; Wiley-VCH Verlag GmbH\& Co. KGaA: Germany:Weinhein, 2014, pp: 131-161.

[15] Fissore, D.; Pisano, R.; Barresi, A. On the use of temperature measurement to monitor a freeze-drying process for pharmaceuticals. Proceedings of IEEE International Instrumentation and Measurement Technology Conference "I2MTC 2017", Torino, Italy, May 22-25, 2017; pp. 1276-1281.

[16] Bosca, S.; Corbellini, S.; Barresi, A.A.; Fissore, D. Freeze-drying monitoringusing a new process analiytical technology: toward a "zero defect" process. Drying Technol. 2013, 31, 1744-1755.

[17] Grassini, S.; Parvis, M.; Barresi, A. A. Inert thermocouple with nanometric thickness for lyophilization monitoring. Trans. Inst. Meas. Control 2013, 62(5), 1276-1283.

[18] Emteborg, H.; Zeleny, R.; Charoud-Got, J.; Martos, G.; Luddeke, J.; Schellin, H., Teipel, K. Infrared thermography for monitoring of freeze-drying processes: instrumental developments and preliminary results. J. Pharm. Sci. 2014, 103, 20882097.

[19] Lietta, E.; Colucci, D.; Distefano, G.; Fissore, D. On the use of IR thermography for monitoring a vial freeze-drying process. J. Pharm. Sci. 2018, 108, 391-398.

[20] Velardi S.A.; Barresi, A.A. Development of simplified models for the freeze-drying process and investigation of the optimal operating conditions, Chem. Eng. Res. Des. 2008, 86, 9-22.

[21] Pearson, K. On Lines and Planes of Closest Fit to Systems of Points in Space. Philos. 
Mag. 1901, 2(11), 559-572.

[22] Hotelling, H. Analysis of a complex of statistical variables into principal components. J. Educ. Psychol. 1933, 24, 417-441, and 498-520.

[23] Nomikos, P.; MacGregor, J.M. Monitoring batch processes using multiway principal component analysis. AIChE J. 1994, 40, 1361-1375.

[24] Wold, S.; Sjöström, M.; Eriksson, L. PLS-regression: a basic tool of chemometrics. Chemom. Intell. Lab. Syst. 2001, 58(2), 109-130.

[25] Nomikos, P.; MacGregor, J.M. Multi-way partial least squares in monitoring of batch processes. Chemom. Intell. Lab. Syst. 1995, 30, 97-108.

[26] Kourti, T. Process analytical technology beyond real-time analyzers: the role of multivariate analysis. Crit. Rev. Anal. Chem. 2006, 36, 257-278.

[27] Van Sprang, E.N.M.; Ramaker, H.J.; Westerhuis, J.A.; Gurden S.P.; Smilde, A.K. Critical evaluation of approaches for on-line batch process monitoring. Chem. Eng. Sci. 2002, 57, 3979-3991.

[28] Rato, T.J.; Rendall, R.; Gomes V.; Chin S.T.; Chiang L.H.; Saraiva, P.; Reis, M.S. A Systematic Methodology for Comparing Batch Process Monitoring Methods: Part I Assessing detection strength. Ind. Eng. Chem. Res. 2016, 55, 5342-5358.

[29] Rato, T.J.; Rendall, R.; Gomes V.; Saraiva, P.; Reis, M.S. A Systematic Methodology for Comparing Batch Process Monitoring Methods: Part II-Assessing Detection Speed. Ind. Eng. Chem. Res. 2018, 57, 5338-5350.

[30] Bharati, M.H.; MacGregor, J.F. Multivariate image analysis for real-time process monitoring and control. Ind. Eng. Chem. Res. 1998, 37, 4715-4724.

[31] Prats-Montalbán, J.M.; De Juan, A.; Ferrer, A. Multivariate image analysis: a review with applications. Chemom. Intell. Lab. Syst. 2011, 107, 1-23.

[32] Duchesne, C.; Liu, J.J.; MacGregor, J.F. Multivariate image analysis in the process industries: A review. Chemom. Intell. Lab. Syst. 2012, 117, 116-128.

[33] De Villiers, J.P.; Leuschner, F.W.; Geldenhuys, R. Centi-pixel accurate real-time inverse distortion correction. Proceedings of SPIE, 2008, 7266:11-1-11-8.

[34] Gonzalez, R.C.; Woods, R.E.; Eddins, S.L. Digital image processing using Matlab. Pearson education, Inc., Upper Saddle River, New Jersey., 2004. 
[35] Colucci, D.

[36] Kourti, T. Application of latent variable methods to process control and multivariate statistical process control in industry. Int. J. Adapt. Control Signal Process. 2005, 19, 213-246.

[37] Kourti, T. Multivariate dynamic data modeling for analysis and statistical process control of batch processes, start-ups and grade transitions. J. Chemom. 2003, 17, 93109.

[38] Camacho, J.; Picó, J.; Ferrer, A. The best approaches in the on-line monitoring of batch processes: Does the modelling structure matters? Anal. Chim. Acta 2009, 642, $59-68$

[39] Kourti, T.; Nomikos, P.; MacGregor, J.M. Analysis, monitoring and fault diagnosis of batch processes using multiblock and multiway PLS. J. Process Control 1995, 5(4), 277-284.

[40] Patel, S.M.; Doen, T.; Pikal, M.J. Determination of the end point of primary drying in freeze-drying process control. AAPS PharmSciTech 2010, 11(1), 73-84.

[41] Kourti, T.; MacGregor, J.M. Multivariate SPC methods for process and product monitoring. J. Qual. Technol. 1996, 28, 409-428.

[42] Nomikos, P.; MacGregor, J.M. Multivariate SPC charts for monitoring batch processes. Technometrics 1995, 37, 41-59.

[43] Nomikos, P. Statistical process control of batch processes. PhD diss., McMaster University, Hamilton, Ontario, 1995.

[44] Arteaga, F.; Ferrer, A. Dealing with missing data in MSPC: several methods, different interpretations, some examples. J. Chemom. 2002, 16, 408-418.

[45] Arteaga, F.; Ferrer, A. Framework for regression-based missing data imputation methods in on-line MSPC. J. Chemom. 2005, 19, 439-447.

[46] Garcia-Muñoz, S.; Kourti, T.; MacGregor, J.F. Model predictive monitoring for batch processes. Ind. Eng. Chem. Res. 2004, 43, 5929-5941.

[47] Camacho, J.; Picó, J.; Ferrer, A. Bilinear modeling of batch processes. Part II: a comparison of PLS soft-sensors. J. Chemom. 2008, 22, 533-547

[48] Folch-Fortuny, A.; Arteaga, F.; Ferrer, A. PLS model building with missing data: New algorithms and a comparative study. J. Chemom. 2017, 31, e2897. https://doi.org/10.1002/cem.2897. 
[49] Bharati, M.H.; MacGregor, J.F. 2000. Texture analysis of images using Principal Component Analysis. Proceeding of SPIE/Photonics Conference on Process Imaging for Automatic Control, Boston, 2017; pp 27-37.

[50] Bellows, R.J.; King, C.J. Freeze-drying of aqueous solutions: Maximum allowable temperature. Cryobiology 1972, 9(6), 559-561.

[51] Tsourouflis, S.; Flink, J.M.; Karel, M. Loss of structure in freeze-dried carbohydrates solutions: effect of temperature, moisture content and composition. J. Sci. Food and Agric. 1976, 27(6), 509-519.

[52] Westerhuis, J.A.; Kourti, T.; MacGregor, J.M. Analysis of multiblock and hierarchical PCA and PLS models. J. Chemom. 1998, 12, 301-321.

[53] Prats-Montalbán, J.M.; Ferrer, A. Statistical process control based on Multivariate Image Analysis: a new proposal for monitoring and defect detection. Comput. Chem. Eng. 2014, 71, 501-511.

[54] Camacho, J.; Picó, J.; Ferrer, A. Multi-phase analysis framework for handling batch process data. J. Chemom. 2008b, 22, 632-643.

[55] Wold, S. Cross-validatory estimation of the number of components in factors and principal components models. Technometrics 1978, 20, 397-405. 


\section{List of Tables}

Table 1. Summary of the experimental operating conditions used and classification of the single batches into the training and the test set.

Table 2. Quality variable matrix Y used inside the PLS based fault detection algorithm. 


\section{List of Figures}

Figure 1. Segmentation of the rgb images for the texture analysis. A: detection of the vials using the Hough transform; B: segmented area; the texture analysis is performed on the pixels of the gray rectangle corresponding to the product inside the vials.

Figure 2. Example of the control charts obtained using the PCA based MSPC algorithm when only the features obtained from the thermal images are used. Gray line: batch 12 , NOC observation; dark gray line: batch 6 , fault detected by both the control charts; light gray line: batch 9 , fault detected only by the SPEI control chart. Black thick line: tuned upper control limits (UCL).

Figure 3. Example of the control charts obtained using the PLS based MSPC algorithm when only the features obtained from the thermal images are used. Gray lines: batch 14, NOC; dark gray lines: Batch 8 (fault); gray line: batch 9, fault detected only by the SPEI control chart. Black thick line: tuned upper control limits (UCL).

Figure 4. Example of the control charts for the quality variables obtained using the PLS based MSPC algorithm. Solid line: on line estimation of the quality variable; dashed lines: 99.5\% control upper and lower limit. Black triangles: expected value. 
Figure 5. Comparison of the classification performances of the PCA based and PLS based MSPC algorithms for different number of principal components extracted. Only the variables obtained from the thermal images are used. $\mathbf{\Delta}$ : PCA, $\mathbf{\square}$ : PLS.

Figure 6. Plot of the additional variables obtained from the PLC of the equipment. A: Temperature of the technological fluid used to provide heat to the product; B: Temperature at the condenser surface; C: Pirani-Baratron pressure ratio; D: chamber pressure measured by the thermal conductive Pirani gauge.

Figure 7. Rgb textural analysis. A: thermal image of batch 7 after $31 \mathrm{~h}$ of primary drying. B: rgb image corresponding to the thermal image. C: Average skewness calculated on a mobile window $3 \times 3$ pixels for all the 10 batches.

Figure 8. Classification performance of the PCA based (A) and PLS (B) based MSPC algorithms with and without additional variables and for different block scaling approaches. $\boldsymbol{\nabla}$ : No additional variable in the $\underline{\mathbf{X}}$ matrix; $\mathbf{\square}:$ Thermal and additional variable without any block scaling $(\mathrm{N}) ; \boldsymbol{\Delta}$ : Thermal and additional variables scaled at unitary variance (U); : Inverse scaling of the two block of data (I).

Figure 9. Cross-validation index for the PLS algorithm with and without additional variables and for different block scaling approaches. $\mathbf{\nabla}$ : No additional variable in the $\underline{\mathbf{X}}$ matrix; 
$\mathbf{\square}$ :Thermal and additional variable without any block scaling $(\mathrm{N}) ; \boldsymbol{\Delta}$ : Thermal and additional variables scaled at unitary variance (U); 0 : Inverse scaling of the two block of data (I). 
Figure 1
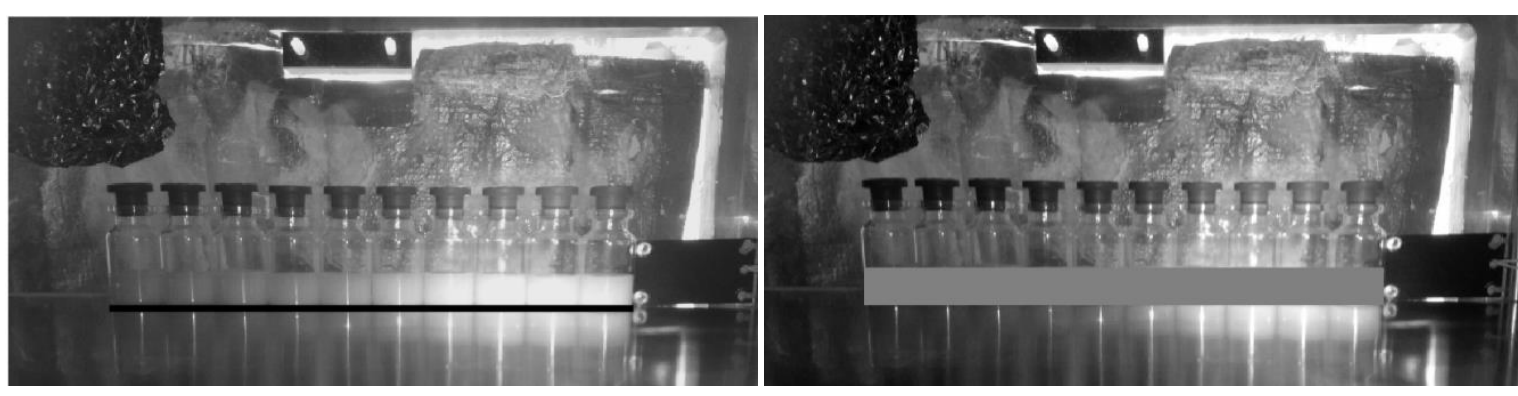

Figure 2
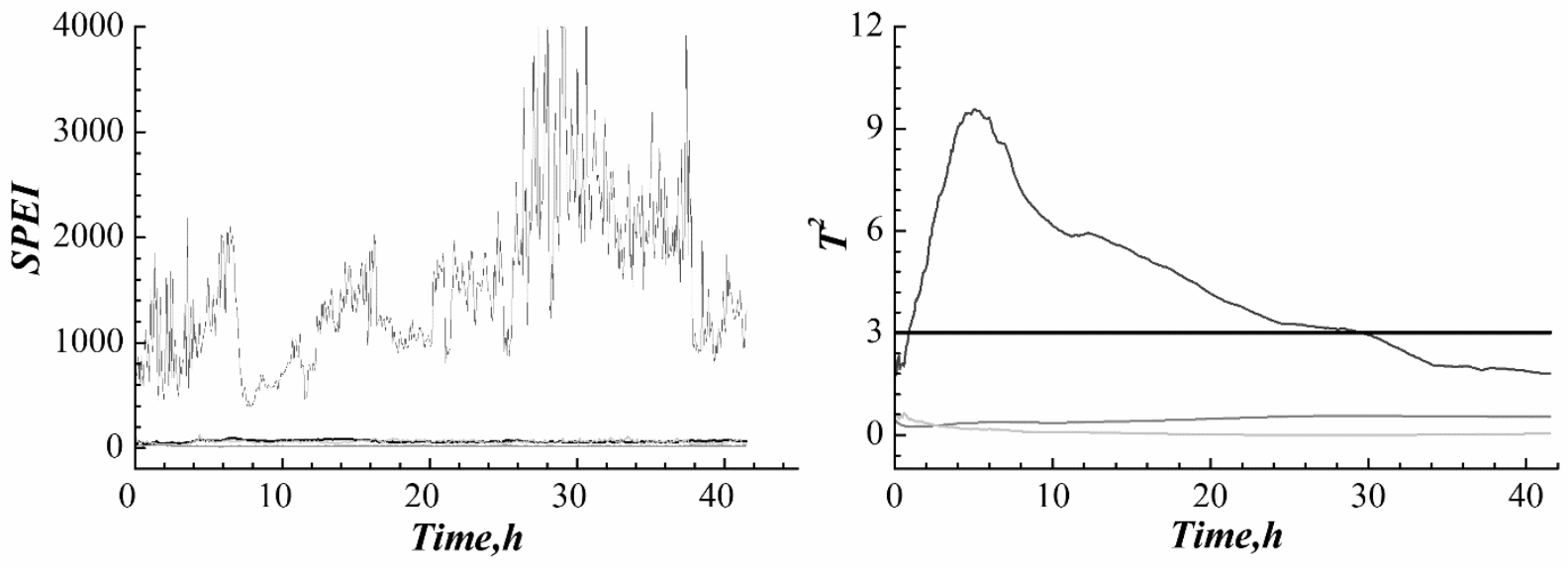
Figure 3
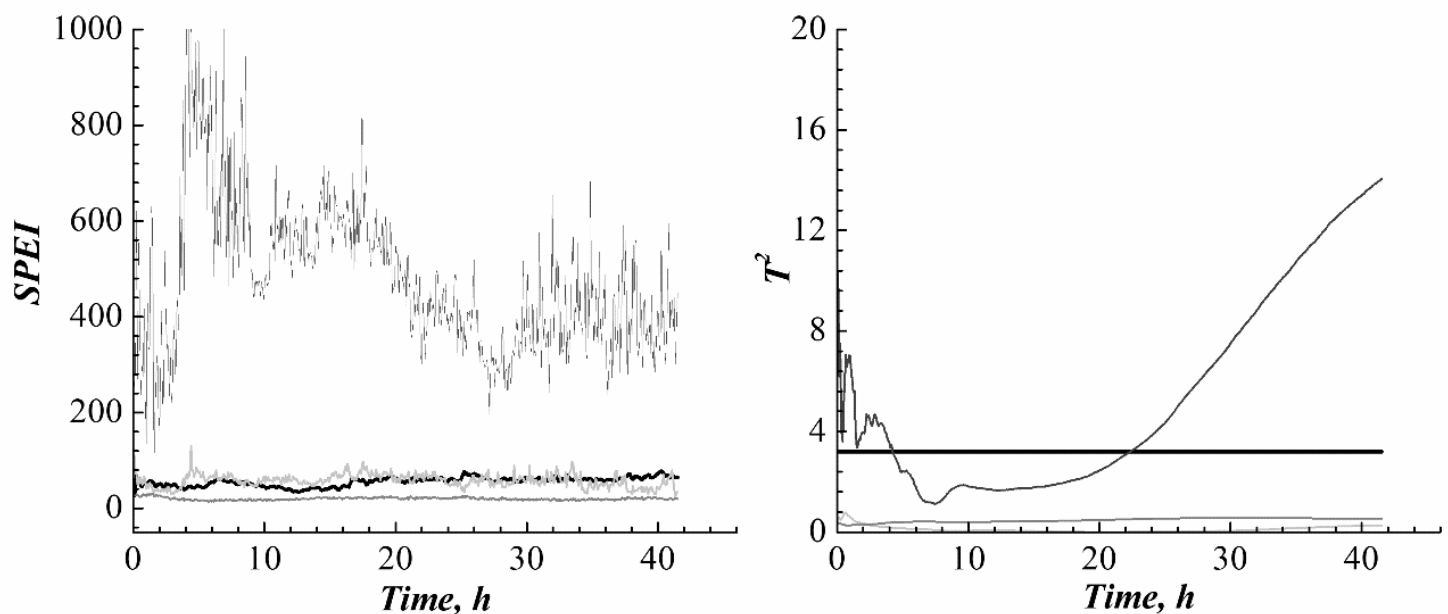
Batch 13
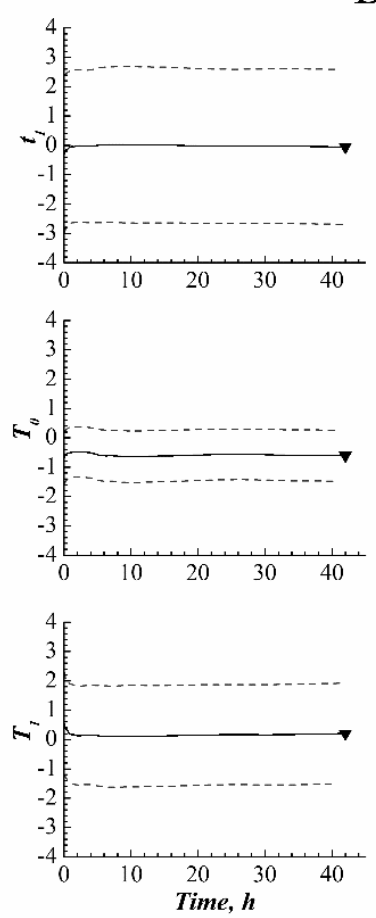
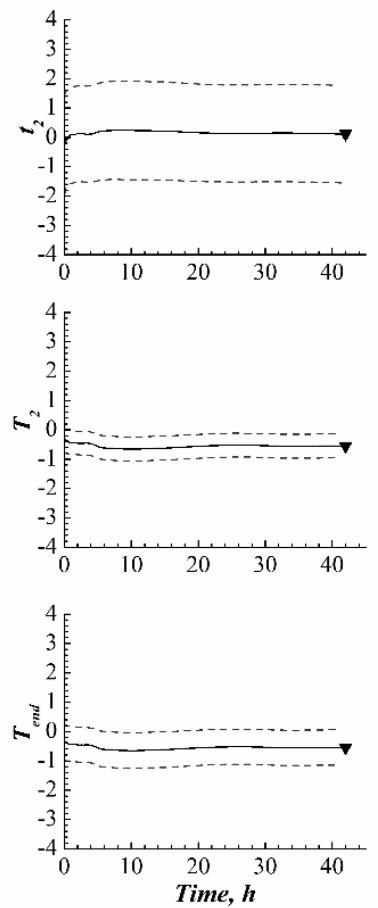

\section{Batch 8}
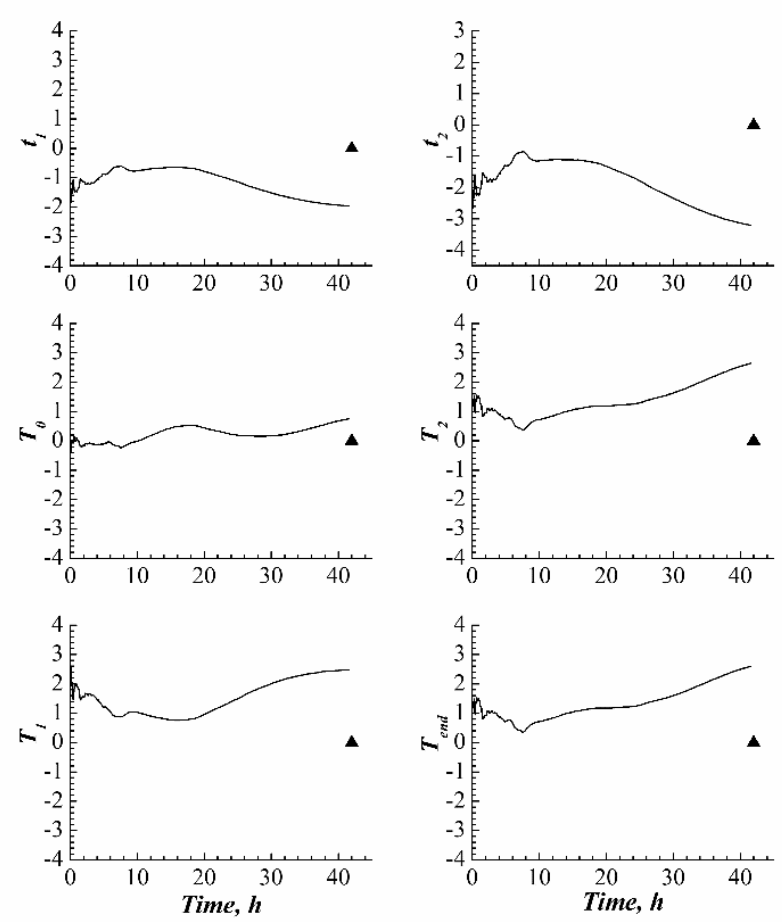
igure 5

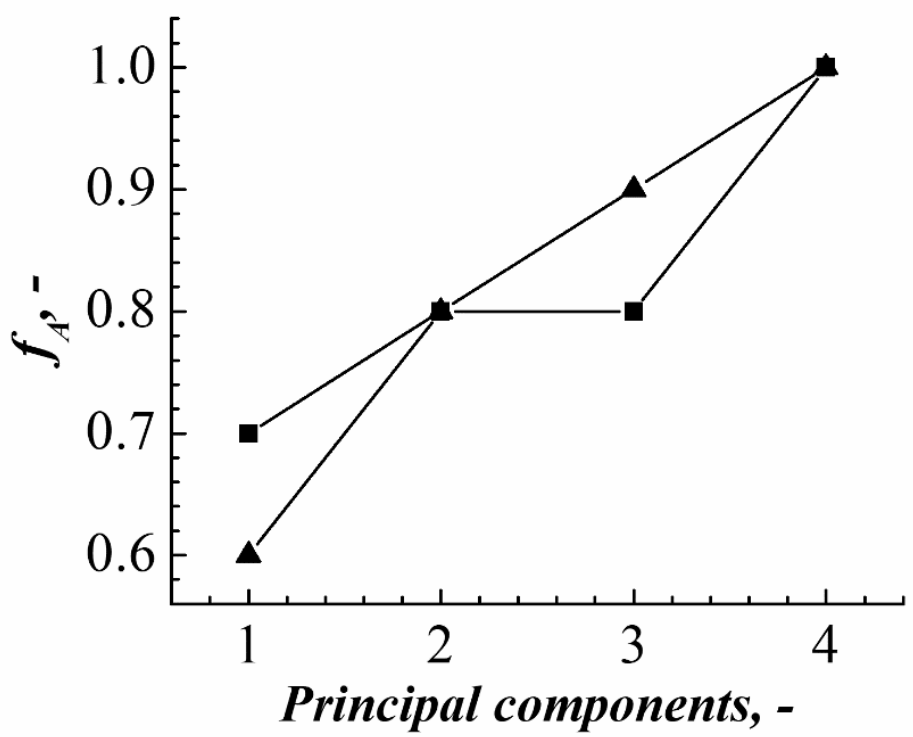


Figure 6
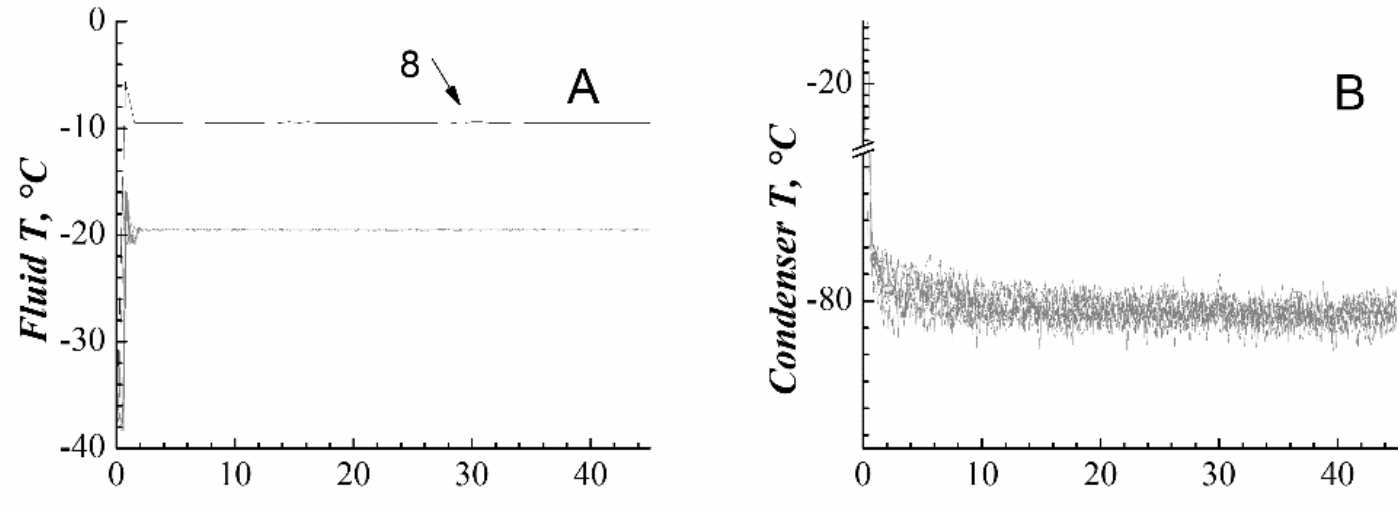

Time, $h$
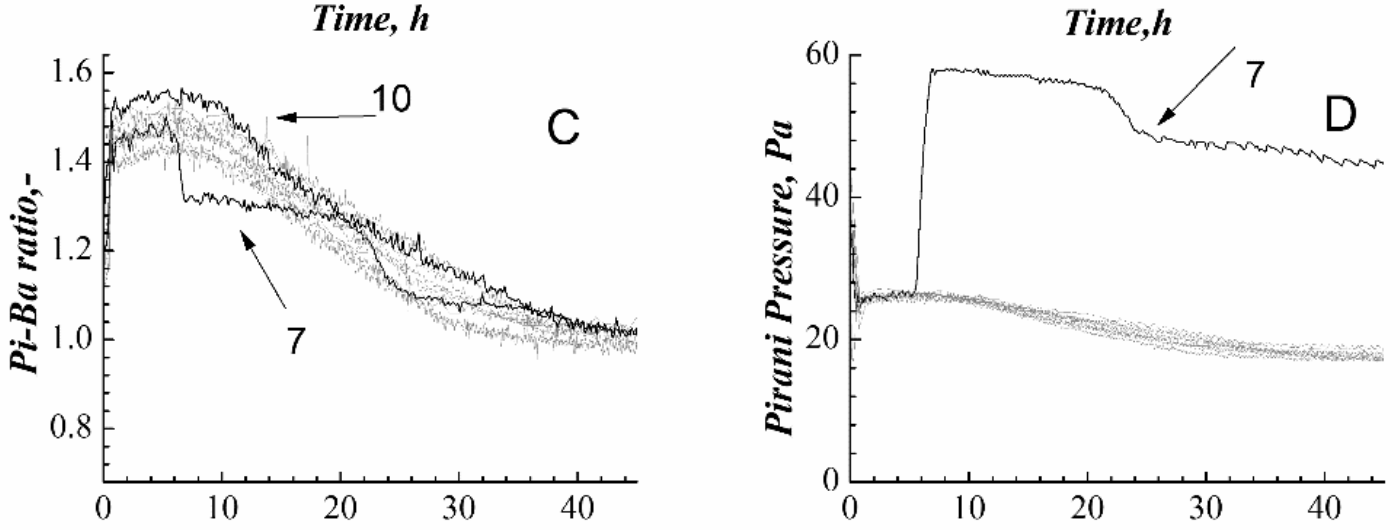

Time, $h$

Time, $h$ 
Figure 7
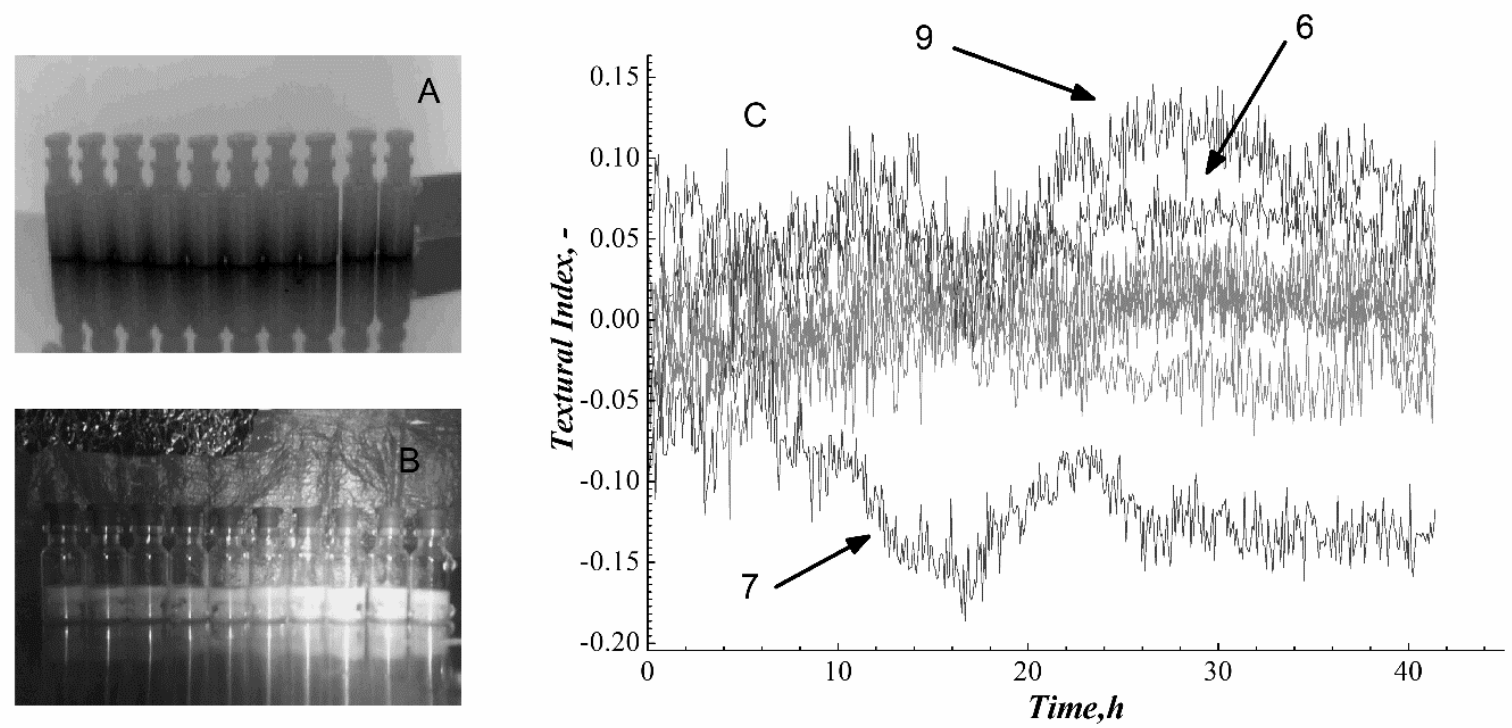

Figure 8
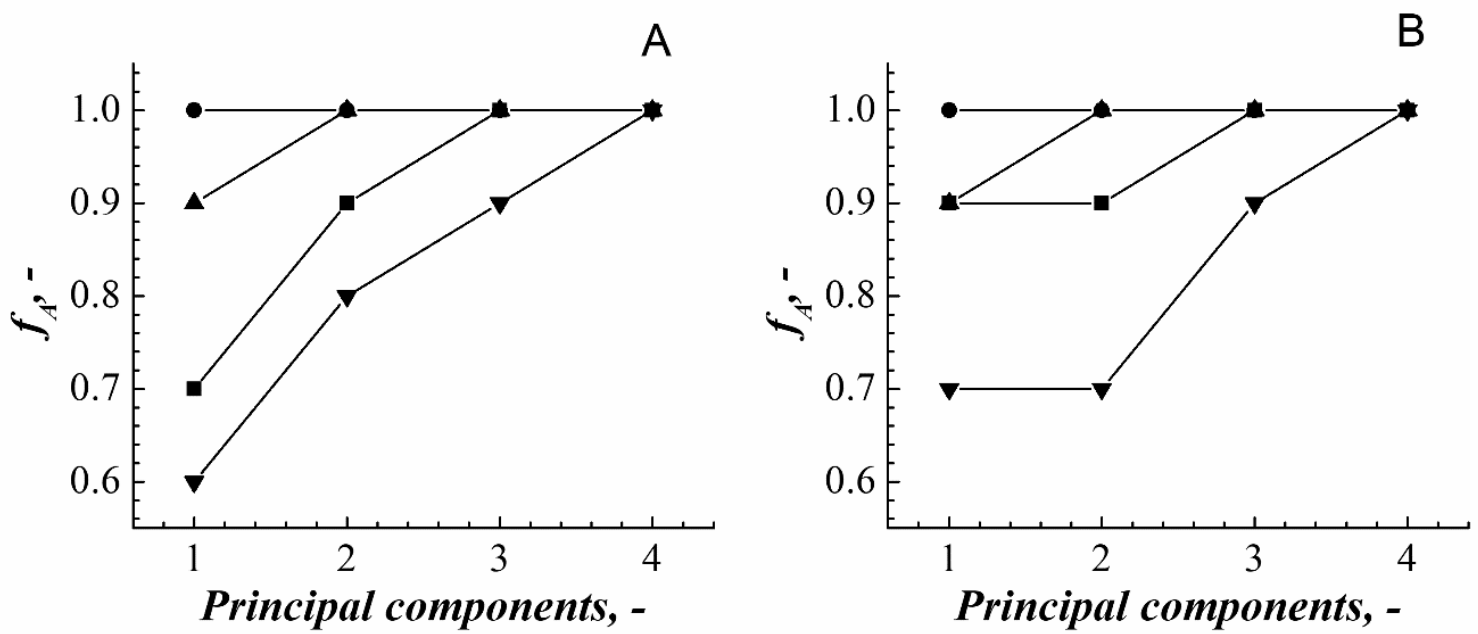
Figure 9

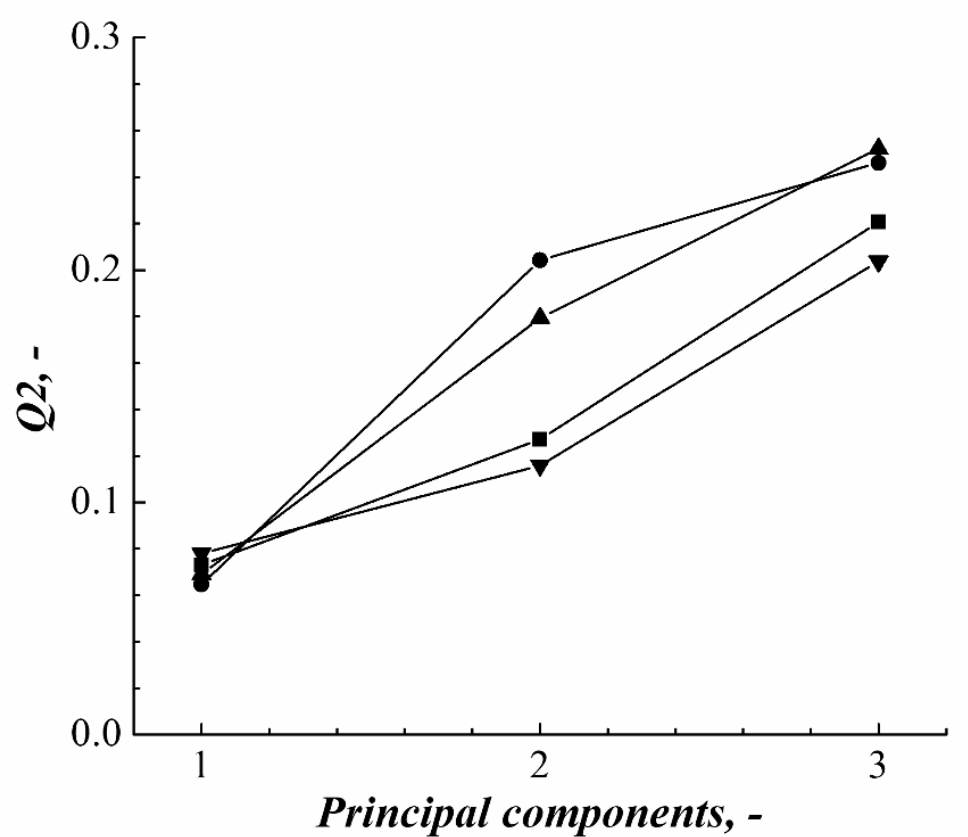


Table 1

\begin{tabular}{|c|c|c|c|c|c|c|c|}
\hline \multicolumn{4}{|c|}{ Batch } & \multicolumn{4}{|c|}{ Operating conditions tested } \\
\hline Number & Type & Observation & Set & Temperature, ${ }^{\circ} \mathbf{C}$ & $\begin{array}{c}\text { Chamber } \\
\text { Pressure, Pa }\end{array}$ & $\%$ Solid & $\begin{array}{c}\text { Volume of } \\
\text { solution, } \mathrm{mL}\end{array}$ \\
\hline 1 & NOC & $1-10$ & Training & -20 & 20 & 10 & 5 \\
\hline 2 & NOC & $11-20$ & Training & -20 & 20 & 10 & 5 \\
\hline 3 & NOC & $21-30$ & Training & -20 & 20 & 10 & 5 \\
\hline 4 & NOC & $31-40$ & Training & -20 & 20 & 10 & 5 \\
\hline 5 & NOC & $41-50$ & Training & -20 & 20 & 10 & 5 \\
\hline \multirow{10}{*}{6} & NOC & 51 & \multirow{10}{*}{ Test } & -20 & 20 & 10 & 5 \\
\hline & NOC & 52 & & -20 & 20 & 10 & 5 \\
\hline & NOC & 53 & & -20 & 20 & 10 & 5 \\
\hline & NOC & 54 & & -20 & 20 & 10 & 5 \\
\hline & NOC & 55 & & -20 & 20 & 10 & 5 \\
\hline & NOC & 56 & & -20 & 20 & 10 & 5 \\
\hline & Fault & 57 & & -20 & 20 & 10 & 5 \\
\hline & NOC & 58 & & -20 & 20 & 10 & 5 \\
\hline & NOC & 59 & & -20 & 20 & 10 & 5 \\
\hline & NOC & 60 & & -20 & 20 & 10 & 5 \\
\hline 7 & Fault & $61-70$ & Test & -20 & $\begin{array}{l}20, \text { raised to } \\
50 \text { after } 5 \mathrm{~h}\end{array}$ & 10 & 5 \\
\hline 8 & Fault & $71-80$ & Test & -10 & 20 & 10 & 5 \\
\hline \multirow{10}{*}{9} & NOC & 81 & \multirow{10}{*}{ Test } & -20 & 20 & 10 & 5 \\
\hline & Fault & 82 & & -20 & 20 & 10 & 5 (glass piece) \\
\hline & Fault & 83 & & -20 & 20 & 10 & 5 (glass piece) \\
\hline & Fault & 84 & & -20 & 20 & 5 & 5 \\
\hline & Fault & 85 & & -20 & 20 & $\begin{array}{l}0 \text { (pure } \\
\text { water) }\end{array}$ & 5 \\
\hline & Fault & 86 & & -20 & 20 & 10 & 2.5 \\
\hline & Fault & 87 & & -20 & 20 & 10 & 7.5 \\
\hline & NOC & 88 & & -20 & 20 & 10 & 5 \\
\hline & NOC & 89 & & -20 & 20 & 10 & 5 \\
\hline & NOC & 90 & & -20 & 20 & 10 & 5 \\
\hline 10 & Fault & $91-100$ & Test & -20 & 20 & 5 & 5 \\
\hline 11 & NOC & $101-110$ & Test & -20 & 20 & 10 & 5 \\
\hline 12 & NOC & $111-120$ & Test & -20 & 20 & 10 & 5 \\
\hline 13 & NOC & $121-130$ & Test & -20 & 20 & 10 & 5 \\
\hline 14 & NOC & $131-140$ & Test & -20 & 20 & 10 & 5 \\
\hline 15 & NOC & $141-150$ & Test & -20 & 20 & 10 & 5 \\
\hline
\end{tabular}


Table 2

\begin{tabular}{c|cccccc} 
Batch & $\mathbf{t}_{\mathbf{1}}, \mathbf{h}$ & $\mathbf{t}_{\mathbf{2}}, \mathbf{h}$ & $\mathbf{T}_{\mathbf{0}},{ }^{\circ} \mathbf{C}$ & $\mathbf{T} \mathbf{1},{ }^{\circ} \mathbf{C}$ & $\mathbf{T}_{\mathbf{2}},{ }^{\circ} \mathbf{C}$ & $\mathbf{T}_{\text {end }},{ }^{\circ} \mathbf{C}$ \\
\hline 1 & 8.03 & 20.50 & -30.81 & -24.01 & -13.29 & -10.82 \\
\hline 2 & 8.03 & 22.60 & -32.43 & -28.90 & -17.71 & -14.08 \\
\hline 3 & 8.65 & 18.59 & -33.18 & -25.74 & -16.63 & -11.60 \\
\hline 4 & 7.30 & 23.57 & -32.98 & -26.46 & -15.04 & -15.37 \\
\hline 5 & 8.80 & 19.59 & -33.00 & -25.54 & -18.02 & -15.65 \\
\hline 6 & 9.08 & 24.98 & -29.45 & -21.54 & -15.08 & -13.72 \\
\hline 7 & 5.90 & 22.00 & -30.78 & -19.43 & -13.37 & -11.76 \\
\hline 8 & 8.12 & 24.73 & -30.27 & -19.85 & -4.50 & -3.96 \\
\hline 9 & 6.65 & 24.07 & -33.33 & -25.79 & -14.68 & -13.89 \\
\hline 10 & 9.06 & 25.45 & -33.10 & -28.20 & -13.80 & -11.60 \\
\hline 11 & 7.03 & 29.63 & -40.15 & -25.58 & -14.14 & -12.74 \\
\hline 12 & 10.3 & 33.25 & -38.14 & -24.31 & -13.60 & -14.54 \\
\hline 13 & 10.1 & 36.87 & -39.39 & -23.03 & -13.60 & -13.74 \\
\hline 14 & 10.43 & 32.1 & -37.95 & -24.31 & -12.78 & -10.00 \\
\hline 15 & 7.76 & 37.5 & -39.09 & -19.99 & -10.27 & -12.74 \\
\hline
\end{tabular}

